\section{IN-OFFICE BLEACHING IN TETRACYCLINE STAINED TEETH: A CASE STUDY}

\author{
Bashar AKM ${ }^{1}$, Afsar BR ${ }^{2}$, Alam MS ${ }^{3}$
}

\begin{abstract}
:
The aim of the study was to evaluate the efficacy of In-office bleaching protocol in tetracycline stained teeth. Discolored dentition diagnosed as homogenous dark brown tetracycline stained teeth was treated with in office bleaching protocol. Desired bleaching has achieved after six sessions of In-office bleaching protocol and satisfactory whitening was apparent throughout the postoperative two-year observation period.
\end{abstract}

\section{Introduction:}

Bleaching has been universally accepted as a method of lightening discolored teeth but tetracycline-stained teeth bleaching is the most challenging form of tooth lightening. ${ }^{1}$ These intrinsic stains, which cannot be removed with polishing or abrasive mechanisms, may result from the administration of tetracycline or its derivatives during childhood, even prenatally, beginning with the second trimester of pregnancy. ${ }^{2}$ The devastating effect of the medication is displayed by teeth of young people with yellow, brown or blue-grey intrinsic stains of the antibiotic tetracycline after being incorporated into the tooth structure during calcification via complexation with calcium ions. ${ }^{3}$ The color and severity of stains are influenced by the type and duration of tetracycline use and the stage of tooth development at the time the medication was prescribed. ${ }^{2}$

The patients having tetracycline staining were not considered candidates for bleaching even before two decades, but with the recent development of an extended (six months) treatment protocol, ${ }^{4}$ many of these stains can now be eliminated. Although according to Glockner and Haywood, tetracycline stains are rather persistent and using bleaching, discoloration can often only be reduced ${ }^{5,6}$, not be eliminated totally ${ }^{7}$.

When bleaching tetracycline-stained teeth in 1997, Haywood, Leonard and Dickenson, were the first to show that using carbamide peroxide applied in trays and used overnight can be effective. ${ }^{8}$ After then there has been resurgence for the 100year-old in-office bleaching technique using 35\% hydrogen peroxide, with or without light. Now a day, several studies have confirmed the efficacy of combined in-office and home care nightguard bleaching on tetracycline-stained teeth and preferred the protocol. ${ }^{9,}{ }^{10}$ However the efficacy of extended

1. Assistant professor, Dept. of Conservative Dentistry\& Endodontics, BSMMU, Dhaka

2. Dental Surgeon, Sonargoen Upzilla Health complex, Narayangang

3. Professor \& Chairman, Dept. of Conservative Dentistry\& Endodontics, BSMMU, Dhaka in-office bleaching protocol through increased bleaching sessions has not been assessed and needed to be explored.

A patient having tetracycline-stained dentition was treated with extended in-office bleaching protocol up to six bleaching sessions in the department of Conservative dentistry and Endodontics, BSMMU for assessing its whitening efficacy and evaluated postoperatively for two years.

\section{Case Report:}

Miss Popy, young lady of 22 years, presented with the chief complaints of discolored teeth. On intraoral examination, homogenous deep brownish discoloration was evident on upper and lower teeth having more darkening in the cervical regions. Oral hygiene status was average. Drug history indicated tetracycline consumption during the early childhood. Radiographic examination of the dentition was found non-contributory. On the basis of the subjective and objective findings, a tetracyclinestained dentition was diagnosed. Extended in-office bleaching protocol was selected for teeth whitening.

One week after the routine periodontal prophylaxis of scaling and curettage, the teeth were polished with flour based pumice to remove the extrinsic stains. Preoperative shade was determined with the help of a "Vita" shade guide and recorded. Photographs were taken with a digital camera to document the color of the teeth.

At the beginning of the office bleaching, cheek retractors were placed and teeth with adjacent gingival tissues were dried. Resin-based isolation barrier as provided with the product (Polaoffice, SDI), was applied on the gingiva adjacent to the teeth to be treated overlapping $0.5 \mathrm{~mm}$ of enamel and light cured for 30-40 seconds in a fanning motion. Supplied powder and liquid was mixed into a gel as per instruction and applied to cover enamel at least $2 \mathrm{~mm}$ in depth. After application on all the anterior teeth of upper and lower jaws, the bleaching gel on each tooth was light cured for 30 seconds with a composite curing light. After completion of light curing on both anterior segments, the gel was suctioned off, the teeth rinsed and the tooth shade was checked for whitening.

Repetition of the bleaching process and the subsequent whitening assessment was done weekly up to the achievement of the desired whitening. Weekly appointments were scheduled in order to reduce sensitivity of teeth. The patient was advised to keep consumption of dark-colored foods and drinks to a minimum, with colored beverages consumed through a straw during the active bleaching phase. After achieving the desired whitening by the end of sixth in-office bleaching, the patient was available for post bleaching evaluation up to two year. Monthly clinical and radiological evaluations throughout the post-operative two year period showed promising results. 


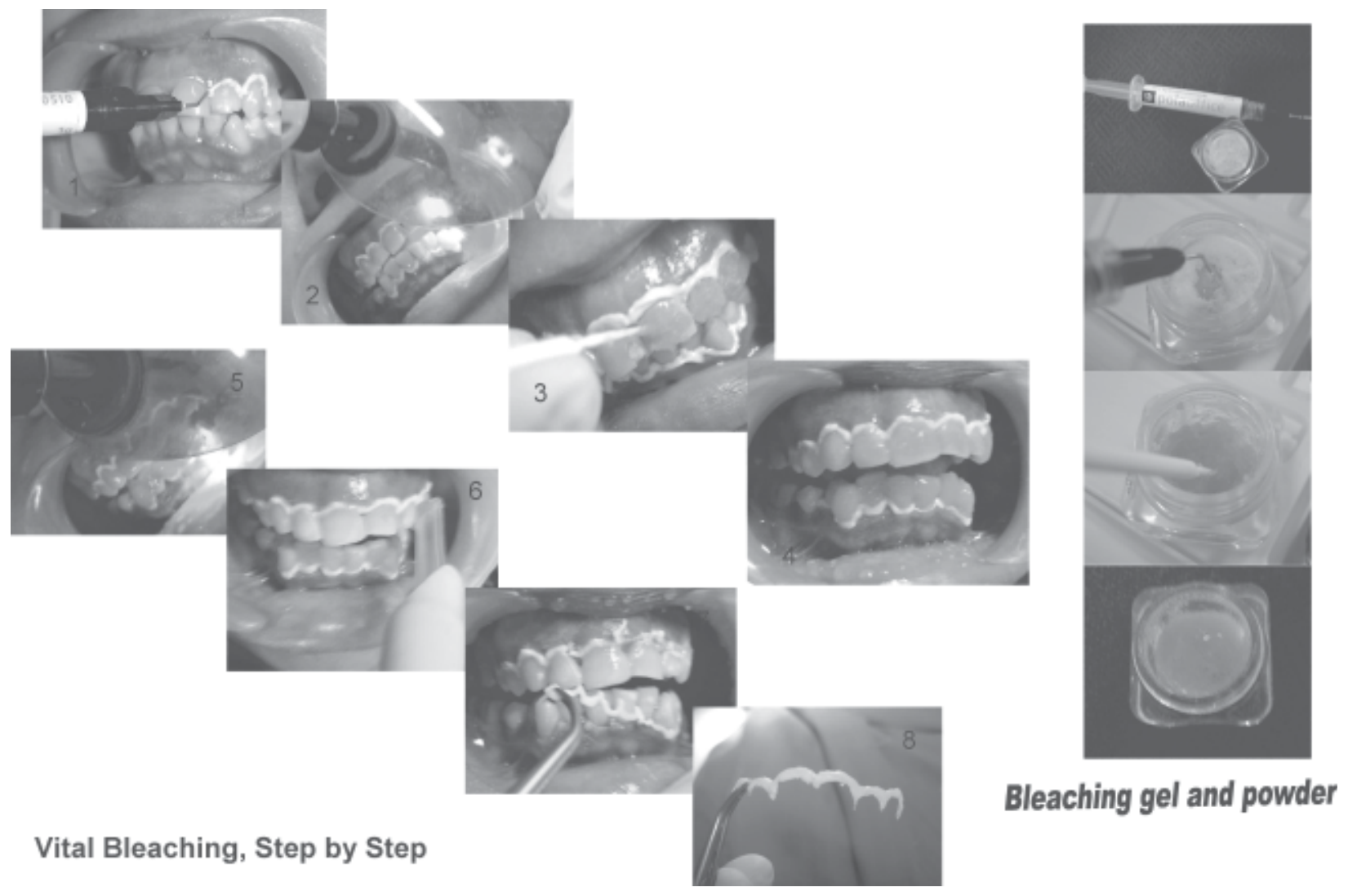

Popy, Vital bleaching (In office power bleaching) for Tetracycline stained teeth

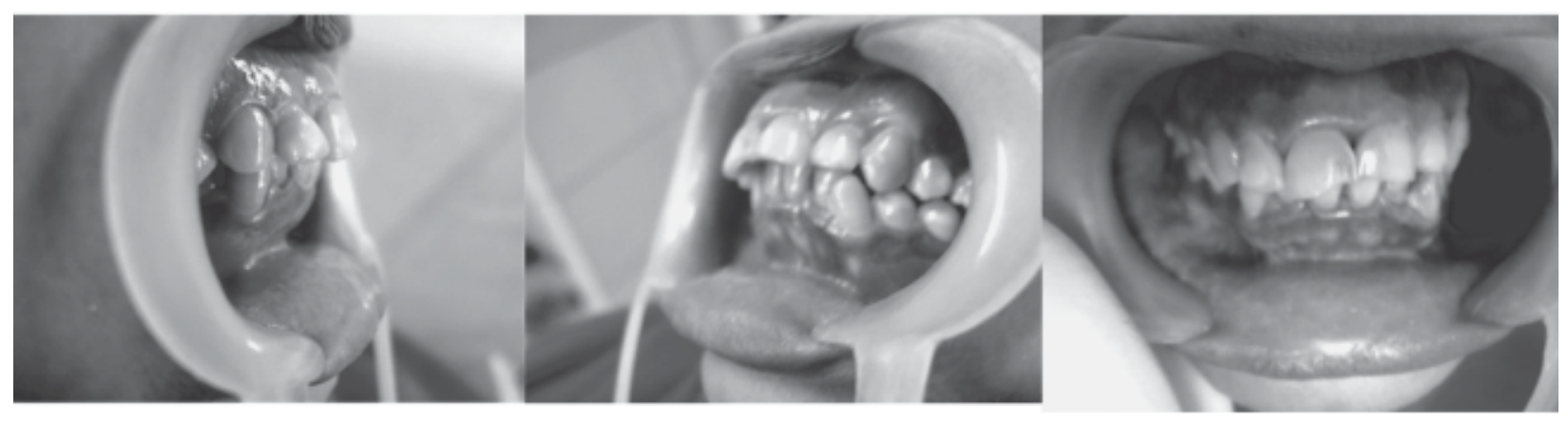

Pre operative view

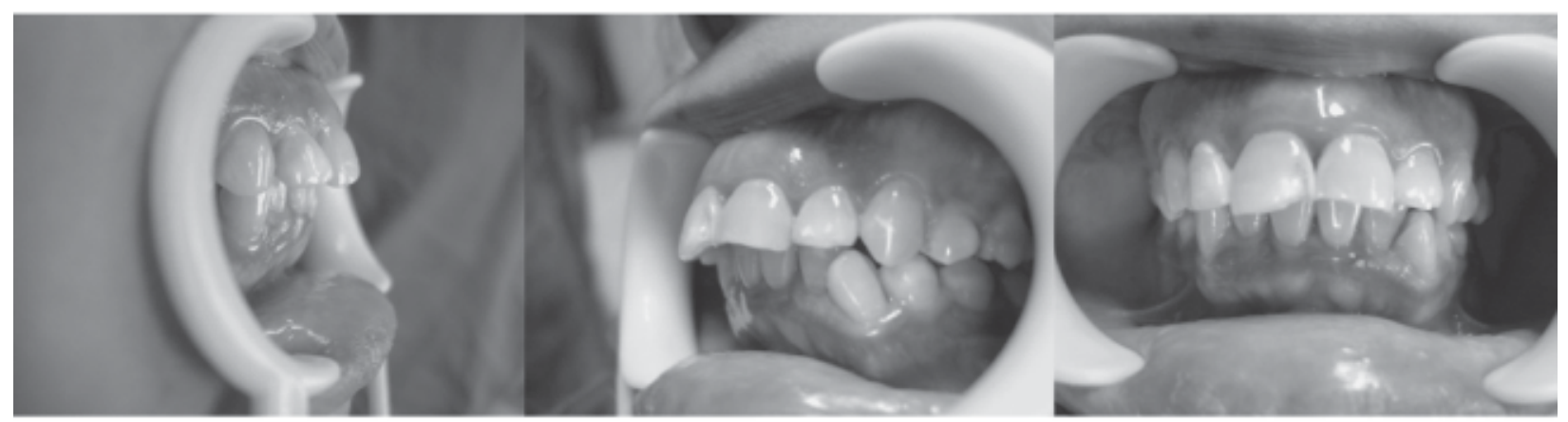

Post operative view 


\section{Discussion:}

Tetracycline staining is the most difficult type of discoloration that was considered resistant to bleaching prior to the introduction of extended treatment protocol for tetracyclinestained teeth in $1997 .{ }^{4}$ With extended treatment protocol, tetracycline-stained teeth may require 1-12 months of overnight bleaching treatment, with an average of 3-4 months for most discolorations. ${ }^{11}$ Although prolonged bleaching can drastically diminish the appearance of severe tetracycline staining but can never fully remove the discoloration. 7,12

For getting a satisfactory result preoperative evaluation of the discoloration status is very important. The intrinsic stain of the antibiotic tetracycline is expressed as the yellow, brown or blue-grey discoloration of teeth. ${ }^{3}$ Among them, the yellow and mild brown discolorations are most responsive and the bluegray and the dark brown discolorations are usually more resistant to bleaching. ${ }^{13}$ Multicolored or "banded" discoloration patterns are not as successfully whitened as other types of discoloration. Although the teeth may exhibit lightening, the banded areas are usually not eradicated completely. ${ }^{14}$ The location of the staining is important, as discoloration at the cervical areas of teeth has a poor prognosis. The statement by Haywood, "The best indicator of a good prognosis is not the severity of discoloration, but the location of the discoloration," 15 is accurate when evaluating tetracycline-stained teeth.

In the present study homogenous deep brownish discoloration, the type that usually responds to several in-office treatments, ${ }^{14}$ was successfully treated using an extended bleaching protocol up to six in-office bleaching sessions without having any post operative relapse for a period of one year. Although for brown discoloration, the combined bleaching protocol consisting of 2 or 3 in-office treatments with 1 to 2 months of at home treatment has been recommended by Dr Goldstein, to achieve desired result, ${ }^{14}$ but, in the present study, dark brown tetracycline discoloration was treated successfully using only in-office bleaching protocol without any at home treatment. Extended bleaching protocol up to six in-office bleaching sessions may be the reason for attaining desired result. New information about tetracycline-stained teeth indicates that stains formerly considered resistant (e.g. the blue-gray and the dark brown) will eventually respond given persistence and an increased number of treatments. ${ }^{13}$ Although in-office bleaching generally requires an average of three application visits for maximum whiteness; ${ }^{14,}$ 16, 17 but to prevent the color relapses ${ }^{18}$ and thus to achieve a long-lasting change during bleaching dark brown discoloration ${ }^{13}$, extended number of in-office bleaching sessions were adopted. For having a complete control and also better patient compliance ${ }^{3}$, instead of using in-office combined home treatment, adopting the extended in-office bleaching sessions alone was found appropriate. To achieve a reasonable result, requirement of these increased number of in-office bleaching sessions also has been mentioned by some authors. $^{14}$

Teeth hypersensitivity, not uncommon in bleaching treatment, was also gained by the patient after $1^{\text {st }}$ bleaching session; but wore off automatically by the application of the bleaching agent week apart and this bears similarity with other recommendations. ${ }^{12}$

\section{Conclusion:}

Although at-home bleaching has increased in popularity, extended in office bleaching protocol may be successfully used for treating tetracycline-stained teeth having homogenous deep brown discoloration. While severe tetracycline staining is not necessarily a contraindication, these cases are difficult to bleach, especially the dark grays and blues; a strict controlled extended in office bleaching protocol may be tried for further evaluation. There are still many indications for in-office bleaching. Where a patient cannot wear bleaching trays, or when patients want to have their teeth bleached quickly and are unwilling to follow the procedural instructions properly at home, in-office bleaching protocol may be the treatment of choice.

\section{References:}

1. Matis BA, Wang Y, Eckert GJ, Cochran MA, Jiang T. Extended Bleaching of Tetracycline-stained Teeth: A 5Year Study. Operative Dentistry 2006; 31(6): 643-651.

2. Kugel G, Aboushala A, Zhou X, Gerlach RW. Daily Use of Whitening Strips on Tetracycline-Stained Teeth: Comparative Results After 2 Months. Compendium / Special Issue 2002; 23(1A): 29-34.

3. Illumine- Professional Bleaching. A technical manual 2001; $1-46$.

4. Haywood VB. Extended bleaching of tetracycline-stained teeth: A case report. Contemporary Esthetics and Restorative Practice 1997;1(1): 14-21.

5. Haywood VB. Nightguard vital bleaching: current concepts and research. JADA 1997; 128 (4): 19S-25S.

6. Glockner K, Ebeleseder K, Städtler P. Das Bleichen von verfärbten Frontzähnen. Schweiz Monatsschr Zahnmed 1997; 107(5): 413-420.

7. Mennito AS. A Simple Guide to Tooth Whitening. The Dental Learning Network 2006; 8-28.

8. Haywood VB, Leonard RH, Dickenson GL. Efficacy of six-months nightguard vital bleaching of tetracycline- 
stained teeth. Journal of Esthetic Dentistry 1997; 9(1): 13-19.

9. Weinberg SP: Bleaching tetracycline stained teeth: a combined approach. Dent Today1997; 8:56-60.

10. Haywood VB, Heymann HO: Response of normal and tetracycline-stained teeth with pulp-size variation to nightguard vital bleaching. J Esthet Dent 1994; 6(3): 109114.

11. Haywood VB. Nightguard Vital Bleaching-Indications and Limitations. US Dentistry 2006; 19-21.

12. Haywood VB. Current Status of Nightguard Vital Bleaching. Compendium 2000; 21(28): S10-S17.

13. Wagner BJ. Whiter Teeth, Brighter Smiles. Access-Special Supplemental Issue 1999; 1-12.
14. Blankenau R, Goldstein RE, Haywood VB. The current status of vital tooth whitening techniques. Compend Contin Educ Dent1999; 20(8):781-796.

15. Haywood VB \& Pohjala R. Bleaching and esthetic bonding of tetracycline-stained teeth. Contemporary Esthetics and Restorative Dentistry 2004; 716-723.

16. Goldstein R. Whitening options: how to choose. Contemp Esthet Restorative Pract 2002; 6(7):12-17. (Interview).

17. Yamaguchi R, Katoh Y. A photocolorimetric study of vital bleaching with Shofu Hi-Lite. J Dent Res 2002; 81:308. (Abstr).

18. Yazici AR, Khanbodaghi A, Kugel G. Effects of an Inoffice Bleaching System (ZOOM ${ }^{\mathrm{TM}}$ ) on Pulp Chamber Temperature In Vitro. The Journal of Contemporary Dental Practice 2007; 8(4): 1-7. 